\title{
Mathematical Modeling of Hemoglobin Release under Hypotonic Conditions ${ }^{*}$
}

\author{
Ivana Pajic-Lijakovic $^{1}$, Branko Bugarski ${ }^{1}$, Milenko Plavsic ${ }^{2}$ \\ ${ }^{1}$ Dept. of Chem. Eng. University of Belgrade, Faculty of Technology and Metallurgy, Belgrade, Serbia \\ ${ }^{2}$ Dept. of Polymer Eng., University of Belgrade, Faculty of Technology and Metallurgy, Belgrade, Serbia \\ Email: iva@tmf.bg.ac.rs
}

Received 2012

\begin{abstract}
Mathematical model is developed to estimate hemoglobin release under hypotonic conditions at microscopic level. The phenomenon of hemoglobin $(\mathrm{Hb})$ release depends on: 1) the dynamics of repeated opening of hemolytic holes and 2) the radial fluctuations of lipid membrane. Both processes are sensitive to the rate of ionic strength decrease within the surrounding medium. Influence of the rate of ionic strength decrease on hemoglobin release is quantified by the model parameters: 1) the specific decrease of erythrocyte radius and 2) the specific decrease of hole radius during single opening time period of hemolytic hole. The prediction of released amount of $\mathrm{Hb}$ influenced by the conductive mechanism is equal to $2.9 \%$. The prediction of total released amount of Hb influenced by the conductive and convective mechanisms is approximately equal to $4 \%$ of the initial amount of Hb within erythrocyte.
\end{abstract}

Keywords: Hemoglobin Transport; Hemolytic Hole; Thermodynamics; Cellular Biology and Engineering; Mathematical Modeling

\section{Introduction}

In last decades, many research groups attempted to design optimal "large-scale" methods for isolation and purification of $\mathrm{Hb}$, with an aim to obtain highly purified, structurally, and functionally preserved molecule, which has been used as a starting material in production of blood substitutes, food additives for prevention of anemia, and/or standards for clinical diagnostics of different molecular variants of Hb. Different procedures of isolation and purification of $\mathrm{Hb}$ from mammals erythrocytes exist [1-2]. Nevertheless, in many of them, first isolation step is osmotic hemolysis. We have optimized osmotic hemolysis of outdated human erythrocytes at macroscopic level ensuring efficiently mixing pattern in bioreactor and regulating the rate of ionic strength decrease within the surrounding solution as reported by Bugarski and Dovezenski [2]. Rate of ionic strength decrease affects rheological response of cell membranes dependently on cell morphology. It influences the processes at microscopic level: the erythrocyte swelling, the repeated osmotic holes opening and on that way the intra-cellular hemoglobin transport and the hemoglobin release through hemolytic holes. The erythrocyte swelling is induced by solvent in-flux driven by total osmotic pressure difference between intra-cellular region and external medium under hypotonic conditions. After in-flux of solvent, in-flux of $\mathrm{Na}^{+}$cations and out-flux of $\mathrm{K}^{+}$cations follows. The formation of a hemolytic hole represents the result of membrane relaxation on swelling caused by solvent in-flux [3-7]. Consequently, such relaxation induces rearrangement of trans-membrane protein band 3 which includes formation of clusters [6]. The hole is bordered by a ring structure of band 3 molecules [4,6].

Deeper insight into phenomenon of osmotic hemolysis oc-

"Ministry of Education and Science of Serbia curred at microscopic level is necessary step for further process optimization. Several investigators have studied complex dynamics of reversible osmotic holes formation through many opening time cycles [3-7]. However, little is reported about the mechanism of intra-cellular $\mathrm{Hb}$ transport depending on the dynamics of holes opening. At the same time, some aspects of the intra-cellular transport phenomena are important for applications of erythrocytes as carriers for various active substances. It is necessary to connect the rheological characteristics of lipid membrane with hemoglobin intra-cellular transport. Main goal of this investigation is the modeling of hemoglobin intra-cellular transport and hemoglobin release in order to predict and optimize osmotic hemolysis at microscopic level based on the proposed model parameters. Model is formulated based on: (1) the local balance equations of $\mathrm{Hb}$ by including (2) the changes of erythrocyte volume and surface described using Young-Laplace equation [7] as well as (3) the changes of osmotic hole area described statistically during the single opening time period of osmotic hole in our previous work [6].

\section{Theoretical Part}

\subsection{Phenomenological Background of the Model}

The phenomenon of $\mathrm{Hb}$ intra-cellular transport under hypotonic conditions is considered. We will formulate the mathematical model for prediction of: 1) the intra-cellular $\mathrm{Hb}$ profiles within erythrocyte during the single opening time period of hemolytic hole and 2) the released amount of Hb. Necessary data for model development are: 1 ) the volume of already swollen erythrocyte decreases $6 \%$ during the single opening time period of hemolytic hole, 2) the released amount of $\mathrm{Hb}$ after three repeated opening time periods of hemolytic hole is about $17 \%, 3$ ) the averaged time period in which hemolytic hole is opened is 
equal to $t_{s}=0.27 \mathrm{~s}$ from Zade-Oppen [8]. Other necessary data for the model development are: 1) the initial averaged volume of swollen erythrocyte [7,9], 2) the radius of reversible opened hemolytic hole [4], 3) the initial intra-cellular concentration of $\mathrm{Hb}$ after erythrocyte swelling [7], 4) Stoke's radius of Hb molecule [10], 5) the effective diffusion coefficient estimated from $[11,12]$. Additional consideration of rheological behavior of $\mathrm{Hb}$ intra-cellular solution from $[13,14]$ is necessary for model development.

Intra-cellular volume fraction of $\mathrm{Hb}$ after swelling and before release is $\Phi=0.289$. Diffusion coefficient of $\mathrm{Hb}$ for infinite dilution is $D_{0}=6.4 \times 10^{-11} \mathrm{~m}^{2} / \mathrm{s}$ as reported by Shuklar [10]. Riveros-Moreno and Wittenberg [11] and Doster and Longeville [12] reported that the effective diffusion coefficient $D_{\text {eff }}$ decreases with the increase of the $\mathrm{Hb}$ concentration as the result of increase the hydrodynamic and potential interactions between $\mathrm{Hb}$ molecules. They reported that $\mathrm{D}_{\text {eff }} / \mathrm{D}_{0} \sim 0.1$ for $\mathrm{Hb}$ volume fraction $\Phi>0.40$. For $\Phi=0.289$, the corresponding value of $D_{\text {eff }}$ is approximately equal to $D_{\text {eff }}=0.2 D_{0}$ based on Doster and Longeville [12] considerations.

The $\mathrm{Hb}$ intra-cellular transport phenomena include conductive and convective mechanisms. The $\mathrm{Hb}$ conductive transport is driven by pressure difference of $\mathrm{Hb}$ between intra-cellular region and external medium. However, the $\mathrm{Hb}$ convective intra-cellular flow represents the consequence of lipid membrane radial fluctuations caused by surface energy changes. It is induced by erythrocyte volume decreases during the single opening time period of hemolytic hole.

\subsection{Model Development}

We formulate the model for considering of the $\mathrm{Hb}$ intra-cellular transport through erythrocyte to the already opened reversible hemolytic hole as well as $\mathrm{Hb}$ release through the reversible hole, under hypotonic conditions. The model could be used for prediction of the intra-cellular transport of some active substances entrapped within erythrocyte for application of cells as drug carriers. The initial time $t=0$ represents the time when the reversible hemolytic hole is opened. We suppose that the initial hole diameter $\mathrm{R}_{\mathrm{H} 0}$ is much higher than the Stoke's radius of $\mathrm{Hb}$ molecule. The final time for modeling consideration is $\mathrm{t}=\mathrm{t}_{\mathrm{s}}$ (the time for which hemolytic hole is closed). It represents the equilibrium time for the single relaxation process of the membrane. Before the model development, it is necessary to estimate the erythrocyte volume $\mathrm{V}_{\mathrm{e}}(\mathrm{t})$. The initial volume of erythrocyte after swelling under hypotonic conditions is $\mathrm{V}_{\mathrm{e} 0}=4 / 3 \pi \mathrm{R}_{\mathrm{e} 0}{ }^{3}$ (where $R_{e 0}$ is the erythrocyte radius at $t=0$ ). The volume decreases during the time period as the result of $\mathrm{Hb}$ out-flow. It is expressed as: $V_{e}=4 / 3 \pi R_{e}(t)^{3}$. The changes of erythrocyte volume and surface during the time period for which hole is opened is considered as the set of the equilibrium states and described using Young-Laplace equation as reported by Delano [7] as $\mathrm{dA}_{\mathrm{e}}(\mathrm{t}) \gamma=\mathrm{dV} \mathrm{V}_{\mathrm{e}}(\mathrm{t})\left(\mathrm{P}_{\text {in }}(\mathrm{t})-\mathrm{P}_{\text {out }}(\mathrm{t})\right.$ ) (where $\mathrm{A}_{\mathrm{e}}(\mathrm{t})$ is the erythrocyte surface, $\gamma$ is the surface tension of erythrocyte membrane). The interior and exterior pressures are expressed using van't Hoff's Law as: $\mathrm{P}_{\text {in }}(\mathrm{t})=\mathrm{RT} \varphi_{\text {in }} \mathrm{C}_{\text {in }}(\mathrm{t})$ and $\mathrm{P}_{\text {out }}(\mathrm{t})=\mathrm{RT} \varphi_{\text {out }} \mathrm{C}_{\text {out }}(\mathrm{t})$ (where $\mathrm{R}$ is the universal gas constant, $\mathrm{T}$ is temperature, $\varphi_{\text {in }}$ and $\varphi_{\text {out }}$ are the averaged intracellular and external osmotic coeffi- cients respectively; $\mathrm{C}_{\text {in }}(\mathrm{t})$ and $\mathrm{C}_{\text {out }}(\mathrm{t})$ are the intra-cellular and external solute concentrations respectively). The radius of erythrocyte $R_{e}(t)$ is expressed as $R_{e}(t)=2 \gamma / \Delta P(t)$ (where the total pressure difference is $\left.\Delta \mathrm{P}(\mathrm{t})=\mathrm{P}_{\text {in }}(\mathrm{t})-\mathrm{P}_{\text {out }}(\mathrm{t})\right)$. Intra-cellular solute concentration for intact cell is equal to $289 \mathrm{~mol} / \mathrm{m}^{3}$ [7]. After swelling, such concentration decreases up to $C_{\text {in }}(0)=138.8$ $\mathrm{mol} / \mathrm{m}^{3}$. During the single opening time period of hemolytic hole, the erythrocyte volume decreases about $6 \%$. After three repeated opening time periods averaged content of $\mathrm{Hb}$ is $83 \%$ of its initial value as reported by Zade-Oppen [8]. Accordingly, the averaged value of the released $\mathrm{Hb}$ content should be less than $10 \%$ per single opening time period of hemolytic hole. For such preliminary condition, the intra-cellular solute concentration should be $C_{\text {in }}(t)=147.5 \mathrm{~mol} / \mathrm{m}^{3}$. We suppose that the volume of external solution is much higher than the volume of single erythrocyte. It indicates that the external solute concentration $\mathrm{C}_{\text {out }}(\mathrm{t}) \sim$ const as well as $\mathrm{P}_{\text {out }}\left(\mathrm{C}_{\text {out }}(\mathrm{t})\right) \sim$ const. The total pressure difference increases during the single opening time period of hemolytic hole as the result of the intra-cellular solute concentration $\mathrm{C}_{\mathrm{in}}(\mathrm{t})$ increase. We simplified the differential changes of the total pressure difference as the linear phenomenon: $\Delta \mathrm{P}(\mathrm{t})=\Delta \mathrm{P}(0) \mathrm{e}^{\mathrm{kt}}$ (where the model parameter $\kappa$ quantified the specific increase of the total pressure difference). The radius of erythrocyte is formulated as: $\mathrm{R}_{\mathrm{e}}(\mathrm{t})=\mathrm{R}_{\mathrm{e}}(0) \mathrm{e}^{-\mathrm{kt}}$. The model parameter $\kappa$ (the specific decrease of erythrocyte radius during single opening time period of hemolytic hole) is equal to $\kappa=1 / t_{s} \ln \left(R_{e}(0) / R_{e}\left(t_{s}\right)\right)$. It depends on the rate of ionic strength decrease within the surrounding medium.

We consider the one-dimensional axial $\mathrm{Hb}$ transport through erythrocyte to the opened hemolytic hole along $\mathrm{x}$-axe. The balance equation for the $\mathrm{Hb}$ transport is expressed as:

$$
\frac{\partial c_{f}(x, t)}{\partial t}=-\frac{\partial}{\partial x}\left[U_{x}(x, t) c_{f}(x, t)\right]+D_{e f f} \frac{\partial^{2} c_{f}(x, t)}{\partial x^{2}}
$$

where $x$ is the axial coordinate such that $x €\left[0,2 R_{e}(t)\right], t$ is time such that $t €\left[0, t_{s}\right], c_{f}(x, t)$ is the local $\mathrm{Hb}$ concentration in the erythrocyte, $D_{\text {eff }}$ is the effective diffusion coefficient and $U_{x}(x, t)$ is the axial velocity. We suppose that the local radial $\mathrm{Hb}$ concentration is constant within the volumetric increments $\Delta \mathrm{V}(\mathrm{x}, \mathrm{t})$ $=A_{x}(x, t) d x$ (where $A_{x}(x, t)$ is the local cross section in axial direction). The $\mathrm{Hb}$ concentration changes along $\mathrm{x}$-axis as the result of $\mathrm{Hb}$ release. Axial velocity is formulated based on the equation of continuity as:

$$
U_{x}(x, t)=U_{r}(x, t) \frac{A_{r}(x, t)}{A_{x}(x, t)}
$$

where $U_{r}(x, t)$ is the radial velocity, equal to $U_{r}(x, t)=\partial h(x, t) / \partial t$, $\mathrm{h}(\mathrm{x}, \mathrm{t})$ is the local radial distance such that $\mathrm{h}(\mathrm{x}, \mathrm{t})=2\left[\mathrm{R}_{\mathrm{e}}(\mathrm{t})^{2}-\right.$ $\left.\left(R_{e}(t)-x\right)^{2}\right], A_{r}(x, t)$ is the surface increment equal to $A_{r}(x, t)=$ $h(x, t) \pi d x$. In that way changes of $h(x, t)$ induce membrane radial fluctuations. The local cross section in axial direction is equal to $A_{x}(x, t)=1 / 4 h(x, t)^{2} \pi$. The molar Hb out-flow through the reversible hemolytic hole to the external hypotonic medium is expressed as:

$$
\frac{\partial n(t)}{\partial t}=\left[U_{x}(x, t) c_{f}(x, t)-D_{e f f} \frac{\partial c_{f}(x, t)}{\partial x}\right]_{x=2 R_{e}(t)} A_{H}(t)
$$


where $n(t)$ is the number of $\mathrm{Hb}$ moles which escape through the hole. The surface area of hemolytic hole $\mathrm{A}_{\mathrm{H}}(\mathrm{t})$ decreases during the time period $t €\left[0, t_{s}\right]$ (where $t_{s}$ is the time such that $A_{H}\left(t_{s}\right)=0$ ). The shape of the osmotic hole is nearly circular. Changes of the area of the hemolytic hole have been formulated as [6]:

$$
A_{H}(t)=A_{H 0} e^{-\lambda_{H} t}
$$

where $A_{H 0}$ is the initial surface area of the reversible hemolytic hole expressed as $\mathrm{A}_{\mathrm{H} 0}=\mathrm{R}_{\mathrm{H} 0}{ }^{2} \pi$ ( $\mathrm{R}_{\mathrm{H} 0}$ is the initial radius of hole) while $\lambda_{\mathrm{H}}$ is the specific decrease of hole radius equal to $\lambda_{\mathrm{H}}=1 / \tau_{\mathrm{rH}}$ (where $\tau_{\mathrm{rH}}$ is the relaxation time for process of hole closing). The model parameter $\lambda_{\mathrm{H}}$ strongly depends on the rate of ionic strength decrease within the surrounding medium. We introduce the first approximation for the value of the relaxation time of the hemolytic hole as $\tau_{\mathrm{rH}} \sim \mathrm{t}_{\mathrm{s}} / 3$ which corresponds to the boundary condition $\mathrm{A}_{\mathrm{H}}\left(\mathrm{t}_{\mathrm{s}}\right)=0$ [6].

The system of modeling equations (1-4) are solved starting from the initial and boundary conditions:

1. At $\mathrm{t}=0$ for $\mathrm{pH}=7.2 \mathrm{Hb}$ molecules are anions uniformly distributed within erythrocyte. The initial $\mathrm{Hb}$ concentration $\mathrm{c}_{\mathrm{f}}(\mathrm{x}, 0)$ is $c_{\mathrm{f}}(\mathrm{x}, 0)=\mathrm{c}_{0 \mathrm{~s}}$ (where $\mathrm{c}_{0 \mathrm{~s}}=3.5 \mathrm{~mol} / \mathrm{m}^{3}$ ).

2. At $\mathrm{t}=\mathrm{t}_{\mathrm{s}}$ erythrocyte volume is equal to $\mathrm{V}_{\mathrm{e}}\left(\mathrm{t}_{\mathrm{s}}\right)=0.94 \mathrm{~V}_{\mathrm{e} 0 \mathrm{~s}}$.

\section{Results and Discussion}

The modeling considerations should point to the influence of the dynamics of repeated opening hemolytic hole and the radial fluctuations of lipid membrane on hemoglobin intra-cellular transport and hemoglobin release through the model parameters: (1) the specific decrease of erythrocyte radius and (2) the specific decrease of hole radius during single opening time period of hemolytic hole. The model parameters quantify process sensitivity to changes of the ionic strength within the surrounding medium. For high experimental rate of ionic strength decrease the model parameters tend to infinity while for low experimental rate of ionic strength decrease the model parameters tend to zero. For experimental data from Zade-Oppen [8] the specific decrease of erythrocyte radius was equal to $\kappa=0.10 \mathrm{~s}^{-1}$ and the specific decrease of hole radius was equal to $\lambda_{\mathrm{H}}=11.1 \mathrm{~s}^{-1}$. We tried to connect such experimental conditions from Zade-Oppen [8] with $\mathrm{Hb}$ intra-cellular transport and $\mathrm{Hb}$ release. The predicted profiles of $\mathrm{Hb}$ concentration during the single opening time period of hemolytic hole was calculated using (1) based on the experimental data from [4,5,7-12]. The profiles were shown in Figure 1. Such profile represented the total result of both: the convective and conductive mechanisms of transport. For deeper insight into the influence of various mechanisms on $\mathrm{Hb}$ intra-cellular transport we calculated separately the prediction of: (1) the amount of $\mathrm{Hb}$ released as the result of the conductive mechanism and (2) the total amount of $\mathrm{Hb}$ released as the result of both, the convective and the conductive mechanisms. It was shown in Figure 2.

The prediction of released amount of $\mathrm{Hb}$ influenced by the conductive mechanism was equal to $2.9 \%$. The prediction of released amount of $\mathrm{Hb}$ influenced by both mechanisms was approximately equal to $4 \%$ of the initial amount of $\mathrm{Hb}$ within erythrocyte. Zade-Oppen [8] experimentally determined that the averaged amount of $\mathrm{Hb}$ released after three jumps (three repeated opening time periods of hemolytic hole) for single

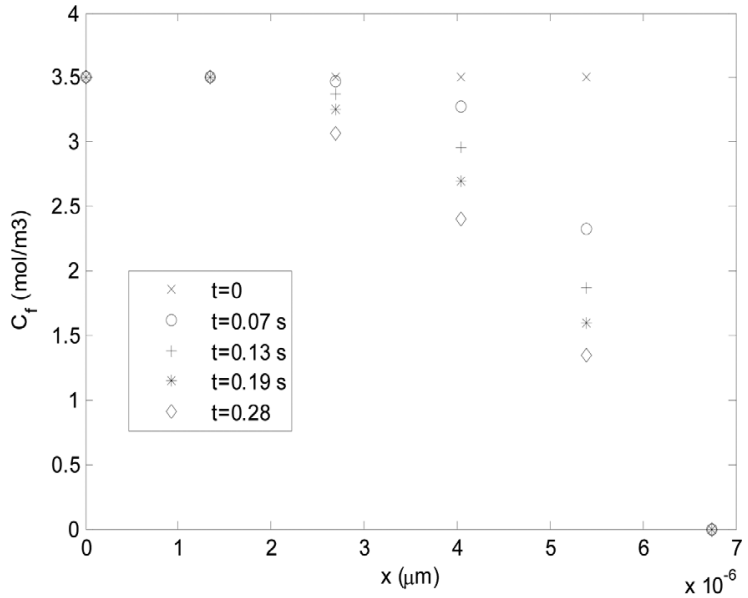

Figure 1. The model prediction of hemoglobin concentration profile within erythrocyte obtained from (1) during the opening time period of hemolytic hole.

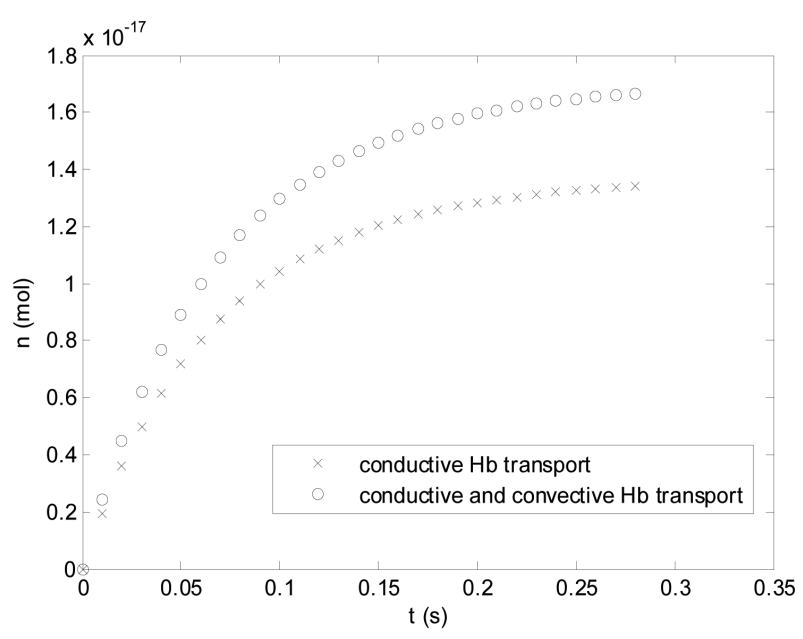

Figure 2. The released amount of hemoglobin obtained from (4) during the opening time period of hemolytic hole.

erythrocyte was equal to $17 \%$ of the initial intra-cellular amount of $\mathrm{Hb}$. The averaged velocity of single cell during jump was equal to $\mathrm{v}_{\mathrm{c}}=6.93 \pm 6.442 \mu \mathrm{m} / \mathrm{s}$ [8]. The corresponding averaged velocity as the result of the conductive transport $D_{\text {eff }} / \Delta x$ was calculated using the proposed model. It was equal to $\sim 9.1$ $\mu \mathrm{m} / \mathrm{s}$. The corresponding averaged velocity for the convective transport was equal to $\sim 1.8 \mu \mathrm{m} / \mathrm{s}$.

\section{Conclusions}

The results of this study point to the influence of the rate of ionic strength decrease within the surrounding medium on the hemoglobin release at microscopic level based on the modeling considerations. The developed model connects the phenomenon of hemoglobin release with the dynamics of repeated opening of hemolytic hole and the fluctuations of lipid membrane. The key model parameters for process optimization are: (1) the specific decrease of erythrocyte radius and (2) the specific decrease of hole radius during single opening time period of hemolytic hole. Higher experimental rate of ionic strength de- 
crease causes undesirable osmotic rupture of cells. For such conditions, the model parameters tend to infinite. On the other side, low experimental rate of ionic strength decrease suppress osmotic hemolysis. For such conditions, the values of model parameters tend to zero. For experimental data from Zade-Oppen [12] the specific decrease of erythrocyte radius is equal to $\kappa=0.10 \mathrm{~s}^{-1}$ and the specific decrease of hole radius is equal to $\lambda_{\mathrm{H}}=11.1 \mathrm{~s}^{-1}$. The corresponding prediction of released amount of $\mathrm{Hb}$ influenced by the conductive mechanism is equal to $2.9 \%$. The prediction of total released amount of $\mathrm{Hb}$ influenced by the conductive and convective mechanisms is approximately equal to $4 \%$ of the initial amount of $\mathrm{Hb}$ within erythrocyte. It is obtained for the initial diameter of the reversible hemolytic hole equal to $3 \mu \mathrm{m}$.

\section{Acknowledgements}

The support by grants (\# III 46010 and \# III46001) from the Ministry of Education and Science, Republic of Serbia is gratefully acknowledged.

\section{REFERENCES}

[1] S.M. Christensen, F. Medina, R.W. Winslow, S.M Snell, A. Zegna, M.A. Marini, "Preparation of human hemoglobin Ao for possible use as a blood substitute” J. Biochem. Biophys. Methods 1988;17, pp. 143-54, 1988.

[2] B. Bugarski, N. Dovezenski, Hemofarm Koncern. Verfahren zur Herstellung von Hemoglobin, Deutsches Patentamt DE 19707508, 2000.

[3] M.R. Leiber, T.L. Steck, "A description of the holes in human erythrocyte membrane ghosts", J. Biol. Chem. 257, pp.11651-11659, 1982.
[4] Y. Sato, H. Yamakose, Y. Suzuki, "Participation of Band 3 Protein in Hypotonic Hemolysis of Hyman Erythrocytes", Biol. Pharmac. Bul. 16(2), pp. 188-194, 1993.

[5] A. Pribush, D. Meyerstein, N. Meyerstein, "Kinetic of erythrocyte swelling and membrane hole formation in hypotonic media", Biochem. Biophys. Acta 1558, pp. 119-132, 2002.

[6] I. Pajic-Lijakovic, V. Ilic, B. Bugarski, M. Plavsic,"The rearrangement of erythrocyte band 3 molecules and reversible osmotic holes formation under hypotonic conditions”, Europ. Biophys. J. 39(5), pp. 789-797, 2010.

[7] M. Delano, "Simple physical constraints in hemolysis", J. Theor. Biol. 175, pp. 517-524, 1995.

[8] A.M.M. Zade-Oppen, "Repetitive cell 'jump' during hypotonic lyses of erythrocytes observed with simple flow chamber", J. Microsc. 192, pp. 54-62, 1998.

[9] G.B. Nash, H.J. Meiselman, "Red Cell and Ghost Viscoelasticity, Effects of Hemoglobin Concentration and In Vivo Aging", Biophys. J. 43, pp. 63-73, 1983.

[10] R. Shuklar, M. Balakrishnan, G.P. Agarwal, "Bovine serum albumin-hemoglobin fractionation: significance of ultra filtration system and feed solution characteristics”, Bioseparation 9, pp. 7-19, 2000.

[11] V. Riveros-Moreno, J.B. Wittenberg, "The Self-Diffusion Coefficients of Myoglobin and Hemoglobin in Concentrated Solutions”, J. Biol. Chem. 247(3), pp. 895-901, 1972.

[12] W. Doster, S. Longeville, "Microscopic Diffusion and Hydrodynamics of Hemoglobin in Red Blood Cells", Biophys. J. 93, pp. 1360-1368, 2007.

[13] A.M. Gennario, A. Luquita, M. Rasia, "Comparison between Internal Microviscosity of Low-Density Erythrocytes and the Microviscosity of Hemoglobin Solutions: An Electron Paramagnetic Resonance Study”, Biophys. J. 71, pp. 389-393, 1996.

[14] D.P. Ross, A.P. Minton, "Analysis of non-ideal behavior in concentrated hemoglobin solution”, J. Molec. Biol. 112, pp. 437-452, 1977. 